\title{
On 'Initial Regime' Cross Flow-Induced Vibration Predictions of Rigid Cylindrical Structures with High Wake Turbulence
}

\author{
AY Elbanhawy ${ }^{1 *}$ and A Turan ${ }^{2}$ \\ ${ }^{1}$ Mechanical Power Department, Faculty of Engineering, Ain Shams University, Egypt \\ ${ }^{2}$ Energy and Multiphysics Group, School of MACE, University of Manchester, United Kingdom
}

\begin{abstract}
Numerical simulations are studied for a circular cylinder undergoing Flow-Induced Vibration (FIV) in highly turbulent cross-flow. The study focuses on wake turbulence and its interaction with the structural response being set in a single degree of Freedom. A novel contribution is presented, where a Reynolds number of 140,000 is numerically studied for FIV-Wake interaction. An unsteady numerical framework is employed for the simulations, incorporating an Arbitrary Lagrangian Eulerian (ALE) method for the associated grid deformation to simulate the coupled motion of the circular cylinder at the starting zone in a typical cylinder-flow response map or what is termed 'initial regime'. Three-dimensional Large Eddy Simulation (LES) and two-dimensional unsteady Reynolds Averaged Navier-Stokes ( $U R A N S$ ) are used to observe the influence of turbulence treatment and domain representation on the simulations carried out. Particular attention is paid towards resolving the large scales of the fluid motion and the inherent coupling of the cylinder's motion towards the associated evolution of the time averaged flow field. Significant changes occur to cylinder hydrodynamics and Reynolds stresses due to flow-induced vibration. Wake mixing is enhanced and kinetic energy production field is qualitatively altered. LES predictions show more accuracy in response and wake dynamics than their $u R A N S$ counterpart which -nonetheless- retain a recognisable merit despite the underlying $u R A N S$ assumptions. The predictions discussed and analysed in detail in the paper compare reasonably with the chosen benchmark tests of the stationary cylinder. Furthermore, it appears that such use of numerical simulations to tackle high Reynolds number FIV is encouraged and suggests that the conclusions outlined regarding the coupled flow-cylinder system potentially provide a valuable insight.
\end{abstract}

\section{Keywords}

Flow-induced vibration, LES, uRANS, Turbulence modelling, Cylinder cross-flow, CFD

\section{Introduction}

Flow-induced vibration is a flow phenomenon that involves multi-disciplinary applications and attracts attention from the scientific community. This describes a fundamental non-linear interaction that occurs between the flow field and the structure across which the flow moves past. Numerical experiments, or Computational Fluid Dynamics (CFD) simulations, have started tackling in earnest the Flow-Induced Vibrations (reffered to as FIV thereafter) as found in Sarpkaya [1], William- son and Govardhan [2], Gabbai and Benaroya [3] and Anagnostopoulos [4]; with focus on circular cylinders. Coverage of a typical flow-excited cylinder response is explained by Blevins [5] with a description of various governing parameters.

It is fair to acknowledge that especially in high subcritical Reynolds numbers and beyond, the range of measurements regarding the wake dynamics in the vicinity of an oscillating cylinder are far from complete. To the authors' knowledge, the open literature offers little

*Corresponding author: AY Elbanhawy, Mechanical Power Department, Faculty of Engineering, Ain Shams University, 1 ElSarayat Street, Abbasia, Cairo, Egypt, E-mail: amr.elbanhawy@eng.asu.edu

Received: March 27, 2017: Accepted: August 02, 2017: Published: August 05, 2017

Copyright: (C) 2017 Elbanhawy AY, et al. This is an open-access article distributed under the terms of the Creative Commons Attribution License, which permits unrestricted use, distribution, and reproduction in any medium, provided the original author and source are credited. 
to know about high subcritical Reynolds numbers turbulence or shear layer transition in the near wake of a flow-excited circular cylinder, let alone other geometries. Dong and Karniadakis [6] attempted a Direct Numerical Simulation (DNS) study to look at forced oscillations implied on a circular cylinder at Re of 10,000, however, the study showed limited results on near wake turbulence. The challenge for turbulence modelling as applied herein -by means of both LES and unsteady RANS - usually inherits a number of problems in its assumptions that are not universally capable of capturing the flow transition details, or the nature of the ensuing non-equilibrium wake turbulence.

A pioneering LES study on a static cylinder was conducted by Breuer [7] for the same Reynolds number discussed in this study. Breuer [7] faced difficulties in obtaining close agreements -in near wake turbulent stresses- with the benchmark experiment of Cantwell and Coles [8] and reported that denser grids may not always yield improved solutions. The numerical implementation of the cylinder vibration in the computational domain adds not but more complications to the flow field resolution. This is one good reason why there are more CFD studies on stationary cylinders than on oscillating ones.

In this study, CFD investigations are presented for a self-excited circular cylinder incorporating low mass and damping in rigid body motion. There is, in general, an established acceptance in the research community that the assumption of rigid body motion is basic and essential to understand the fluid-structure coupling as indicated in the reviews aforementioned. An example of such approach is given by Elbanhawy and Turan [9] where CFD is applied to study a free oscillating cylinder at Reynolds number of 4000 . However, the high -almost critical- Reynolds number studied herein addresses an industrial need that is generally encountered in large diameter marine risers in ocean currents, or in process plants for cross-flow heat exchangers' design.

The present exercise is aimed at examining two different turbulence treatment methods, namely LES and uRANS, compared to adequate quality experimental data. The Reynolds number of the simulated cross-flow over a circular cylinder is 140,000 , which to the authors' knowledge, has not been tested in detail for FIV in the open literature.

While evidences are that coherent vortices are present in the studied high Reynolds number [10], an experimental attempt by Govardhan and Williamson [11] suggests that periodic structures are significantly enhanced by the presence of FIV. Their experiment has shown an increase of the periodic turbulent component for up to $90 \%$ of the total turbulent stresses for the Reynolds num- ber of 3900. As So, et al. [12] confirm in their experiment that cylinder vibration causes insignificant effect to random turbulence in the near wake, the focus on coherent structures in the wake is a reasonable technique to observe the influence of FIV on the near wake.

The influence of Reynolds number on the FIV response has been only looked at relatively recently. Pioneering work of Govardhan and Williamson [13] confirms that maximum amplitude of a cylinder in transverse FIV increases as the Reynolds number increases provided that maximum Reynolds number tested was 33,000. Using CFD, Elbanhawy and Turan [14] confirmed existence of higher amplitudes in high Reynolds number flows. In light of this influence, the present study contributes in two areas: exploring the high subcritical Reynolds number cross flow vibration and attempting to resolve the coherent wake turbulent structures. By virtue of its methodology, the 3D LES study herein captures the flow scales down to the size of the grid filter that is directly decided based on the actual grid size. Alternatively, by its fundamental assumptions, the $2 \mathrm{D} u R A N S$ study is designed to only capture the coherent and large eddies in the solution domain.

It is fair to express an important reservation here however in that $2 \mathrm{D}$ simulations generally suffer from the common drawbacks that previous researchers had 4 encountered Tamura, et al. [15]. Moreover, the approximation implied by the use of $u R A N S$ has its own limits and caution should be exercised during its undertaking as comprehensively reported in Bouhadji, et al. [16]. Owing to the complicated wake dynamics in conjunction with the numerical grid issues arising from the cylinder motion, it is fair to state that CFD studies on FIV are challenging but nevertheless a worthy goal to strive. Notwithstanding the fact that the subject of FIV is an analytically/computationally demanding topic for many industry practitioners, the value that the current effort presents stems from the practicality of numerical simulations incorporating modelled turbulence fields. The ability to extend the numerical simulations to cover geometrically complex oscillating bodies, or even multiple body vibrations, further suggests that more research is needed for FIV applications.

\section{Nomenclature}

$D$ : Cylinder diameter; d: Space dimension; $\mathrm{V}_{\mathrm{r}}$ : Reduced velocity; $f_{n}$ : Cylinder's natural frequency; U, Uin: Mean velocity, Mean Inlet velocity; $f_{\text {osc }}$ : Cylinder's oscillating frequency; $\rho$ : Density of working fluid; $\overline{C d}$ : Time averaged drag coefficient; $u, v, p, t(\mathrm{x}, \mathrm{y})$ velocities, pressure and time respectively; $C^{\prime} d$ : Fluctuating drag coefficient; $u^{\prime}, \tilde{u}, \bar{u}$ Instantaneous, phase averaged and filtered velocity respectively; $\mathrm{L}_{\text {rec }}$ : Recirculation length based on zero time averaged velocity; $y_{c y l .}$ : Cylinder's 
vertical displacement, Cpbase: Base pressure coefficient; $\mu, \mu_{t}$ : Laminar and turbulent viscosity respectively; $\theta_{s}^{0}$ Time averaged separation angle; $v_{\mathrm{tr}}$ Turbulent kinematic viscosity; $C^{\prime} l$ : Fluctuating lift coefficient; $f_{\text {Shed }}$ : Vortex shedding frequency; $\Delta \Phi$ : Phase angle between lift force and the cylinder's displacement; $\delta_{i j}$ : Kronecker Delta; $F$, $F_{\text {fluid }}:$ Force, Fluid force coefficient in transverse direction; $P_{k e-p}$ : Kinetic energy production of the coherent vortical structures; $\dot{y}(t)$ : Time series of the cylinder's velocity; $\mathrm{x}$, $y$ : Horizontal and vertical Cartesian coordinates respectively, $\ddot{y}(t)$ : Time series of the cylinder's acceleration; $\overline{u u}, \overline{v v}, \overline{u v}$ : Time averaged total streamwise, transverse and shear Reynolds stresses; $\overline{\tilde{u} \tilde{u}}, \overline{\tilde{v} \tilde{v}}, \overline{\tilde{u} \tilde{v}}$ : Time averaged periodic streamwise, transverse and shear Reynolds stresses; $C_{l v}$ : Force coefficient in phase with cylinder's velocity; Cla: Force coefficient in phase with cylinder's acceleration.

\section{Objectives and Methods}

Two turbulence treatment methods for CFD, LES and uRANS are explored for FIV in the 'initial regime' at a single reduced velocity; the definition of reduced velocity follows as $V r=U i n=f n D$. The emphasis-contrary to the previous published predictions is on the statistics of resolved coherent flow structures. In this study use is made of the commercial code Fluent [17], a collocated finite volume code, to solve the flow and turbulence equations in Cartesian coordinates. Initial validation for static cylinder wake is made against measurements of Cantwell and Coles [8] and Perrin, et al. [18]. Predictions of Breuer [7] are also used for comparisons where applicable. The implementation of the cylinder's self-excited oscillations is undertaken by an Arbitrary Lagrangian Eulerian (ALE) deforming mesh technique of which predictions are investigated numerically vis-a-vis the benchmark experiment of Brankovic and Bearman [19] and Hover, et al. [20]. Statistical calculations are based on the turbulent unsteady flow triple decomposition as suggested by Reynolds and Hussain [21].

\section{Computational Aspects}

The computations herein are carried out using a cell-centred finite volume method based on a multidimensional linear reconstruction scheme as that described in [9]. For the LES computations, a bounded central differencing scheme is used for the convective fluxes as developed by Leonard [22]. Whereas for the uRANS computations, use is made of a second order upwind-biased scheme to calculate the turbulence scalars as this provides stability for the calculation of turbulent viscosity. A blended central-differencing scheme with upwinding (Quadratic Upstream Interpolation for Convective Kinetics) is employed to discretize the $u R A N S$ equations in two dimensions as applied in Leonard and Mokhtari [23]. This scheme uses a three-point upstream-weighted quadratic interpolation for the cell face values containing the two side cells of the face plus a third cell on the upstream side [17]. To achieve the unsteady time marching, a first order backward-difference implicit time integration scheme is used with a non-dimensional time step of 0.001 and 0.00224 , for the LES and $u R A N S$ cases respectively, achieving a maximum value of Courant number of less than one.

\section{Domain and boundary specification}

The LES simulation has a 3D grid in which the cylinder's span-to-diameter ratio is 2 . The $u R A N S$ simulation has a $2 \mathrm{D}$ grid. Both $L E S$ and $u R A N S$ cases share the same dimensions in the stream wise transverse plane.
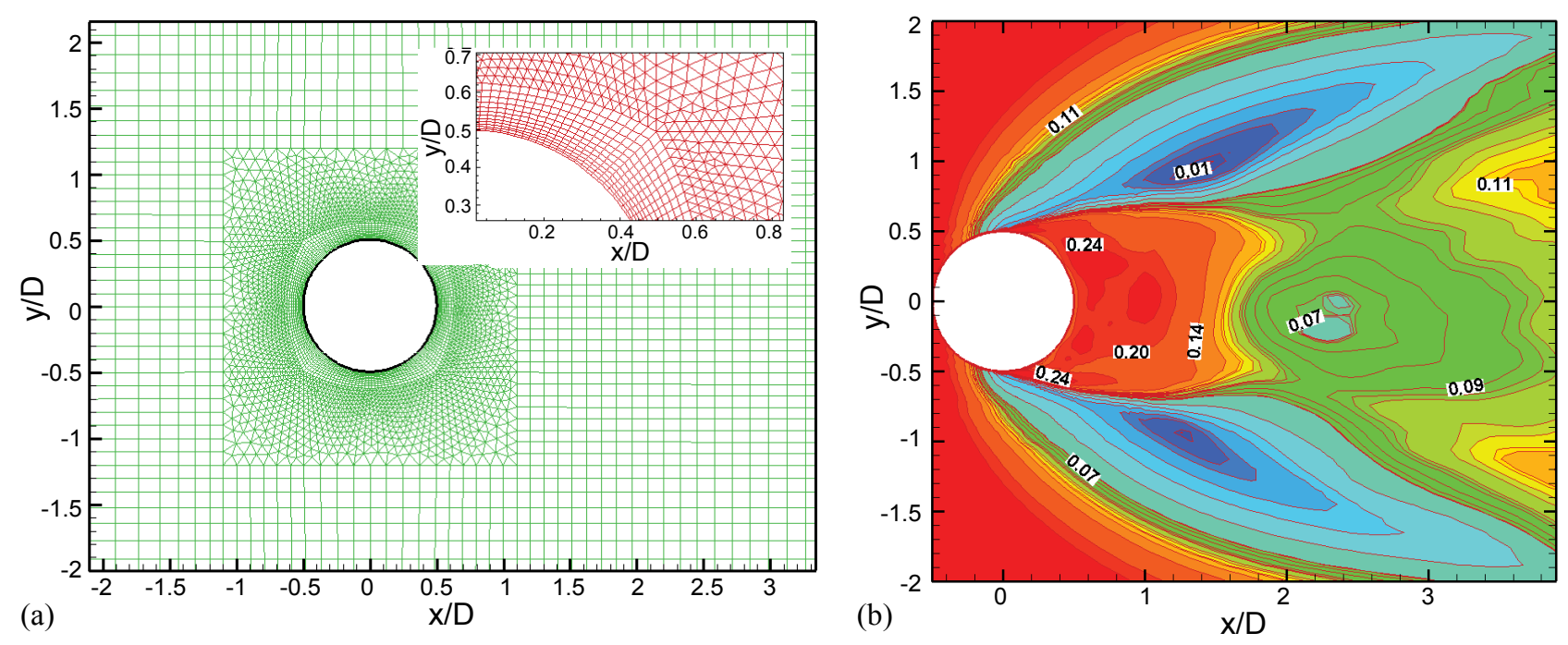

Figures 1: a) Cross-section showing Grid topology; the boundary layer zone is adjacent to a hybrid unstructured zone where the mesh motion occur; b) Contours showing $K . E_{S G S} / K . E_{L E S}$, ratio of subgrid modelled turbulent scales to the resolved scales, made for the coarse LES grid. 
The computational domain in the present work has a rectangular section; the domain extends 3.5 diameters upstream of the cylinder, 8.5 diameters downstream and 4.5 diameters on the top and bottom sides with water as working fluid. This translates into a blockage ratio of $11.1 \%$ and a span-to-diameter ratio of infinity for the $2 \mathrm{D}$ uRANS case.

As shown in Figure 1a, the grid topology comprises structured quadrilateral elements -hexahedral in $3 \mathrm{D}$ - in the boundary layer zone around the circumference. This layer is designed to resolve the laminar sub layer by satisfying a maximum circumferential wall non-dimensional distance $\mathrm{Y}^{+}$of one and a maximum cell size transition ratio of 1.17 in all the tested simulations, hence, eliminating the need for wall functions approximations. In the case of the FIV predictions, the boundary layer zone is conned to move as a single entity in compliance with the cylinder's surface. This enables collection of statistics as the cell indices and sizes are not altered. This quadrilateral/hexahedral structured zone is followed by unstructured triangular/pyramidal elements for what is termed the dynamic mesh zone. The remaining of the domain is meshed with structural quadrilateral/hexahedral cells.

For the 3D LES tests, the a posteriori assessment of the grid filter is shown in Figure $1 \mathrm{~b}$ and shows that the modelled part of turbulent scales reached a maximum of $24 \%$ of the total turbulent fluctuations. This resolution is a function of the Dynamic Kinetic Energy sub-grid scale model used in this analysis. As for the 2D uRANS grids, all constructed grids satisfy a resolution in the wake such that a single cell size is less than one tenth the integral length scale computed by $k^{1.5} / \varepsilon$ where $k$ is the turbulence kinetic energy and $\varepsilon$ is the turbulence dissipation rate. Subsequently, the $u R A N S$ grid resolution is designed to capture the energy containing large scale periodic flow structures. Grid construction data are listed in Table 1.

The inlet section is ascribed a constant uniform velocity. In the LES case, the inlet has no perturbations to allow a fully laminar approaching flow to the cylinder surface. In the $u R A N S$ case, the inlet is ascribed low turbulence intensity of $0.6 \%$ to set a small value of the turbulent viscosity. Also, a no-slip adiabatic condition is specified for the cylinder surface that is assumed to be perfectly smooth. At the outlet boundary, a fixed pressure value is maintained, while all other flow variables are extrapolated from the interior. Additionally, there is no restriction on the flow gradients at the outlet boundary. A symmetry condition is applied at the top and bottom boundaries. In the 3D LES case, the domain sections at each end of the cylinder are specified as symmetry sides leaving no imposition of sectional correlation at either side. While the outlet boundary does not offer treatment for reflections, static-pressure based quantities indicate negligible effect of any reflection, such as spurious fluctuations.

\section{Flow and structure model}

Incompressible Navier-Stokes equations, continuity and turbulence scalars equations are discretized and implicitly solved iteratively by the Fluent solver. In the LES case, use is made of a filtered Navier-Stokes and continuity equations that solve for the resolved flow fluctuations including the effect of Sub Grid Scales (SGS) stresses:

$$
\frac{\partial \bar{u}_{i}}{\partial t}+\frac{\partial \bar{u}_{i} \bar{u}_{j}}{\partial x_{j}}=-\frac{\partial}{\partial x_{j}}\left(\psi_{i j}\right)-\frac{\partial \bar{p}}{\partial x_{i}}-\frac{\partial T_{i j}}{\partial x_{j}}
$$

Where $\left(\psi_{i j}\right)$ is the stress tensor due to molecular viscosity and Tij is the SGS stress defined by

$$
T_{i j} \equiv \rho \overline{u_{i} u_{j}}-\rho \overline{u_{i}} \bar{u}_{j}
$$

The SGS stresses are modelled based on the Dynamic Kinetic Energy SGS Model devised by Kim and Menon [24] in which the turbulent viscosity $\mu_{t}$ is calculated from the SGS kinetic energy $k_{s g s}$ as

$$
\mu_{t}=C_{k} k_{s g s}^{0.5} \Delta f
$$

And the SGS stresses are calculated from

$$
T_{i j}-(2 / 3) k_{s g s} \delta_{i j}=-2 C_{k} k_{s g s}^{0.5} \Delta_{f} \overline{S_{i j}} \text {, }
$$

And $k_{s g s}$ is found by solving its transport equation iteratively with the flow equations,

$$
\frac{\partial \overline{k_{s g s}}}{\partial t}+\frac{\partial \bar{u}_{j} \overline{k s g s}}{\partial x_{j}}=\frac{\partial}{\partial x_{j}}\left(\mu_{t} \frac{\partial k_{s g s}}{\partial x_{j}}\right)-C_{\varepsilon} \frac{k_{s g s}^{1.5}}{\Delta_{f}}-T_{i j} \frac{\partial \bar{u}_{i}}{\partial x_{j}}
$$

\begin{tabular}{|c|c|c|c|c|}
\hline Grid & Cylinder's $1^{\text {st }}$ cell & Wake $X, Y, Z$ elements & Grid Points & Radial cell size increase ratio \\
\hline & $x / D, y / D, z / D$ & & & \\
\hline$R 1$ & $0.82 \%, 0.006 \%$ & 64,65 & 28,626 & 1.135 \\
\hline$R 2$ & $0.065 \%, 0.006 \%$ & 64,65 & 43,232 & 1.109 \\
\hline$R 3$ & $1.9 \%, 0.016 \%$ & 50,56 & 13,115 & 1.165 \\
\hline$R 4$ & $0.96 \%, 0.008 \%$ & 50,64 & 31,686 & 1.165 \\
\hline R5 & $0.96 \%, 0.008 \%$ & 58,135 & 39,918 & 1.165 \\
\hline Coarse LES & $1.3 \%, 0.016 \%, 5.5 \%$ & $70,77,36$ & $1,013,868$ & 1.1 \\
\hline Fine $L E S$ & $0.96 \%, 0.016 \%, 4 \%$ & $125,95,50$ & $2,161,391$ & 1.07 \\
\hline
\end{tabular}

The rate-of-strain tensor $S_{i j}$ is based on the resolved filtered velocity gradients. The filter size $\Delta_{f}$ is equivalent

Table 1: Computational grid structure for sensitivity analysis. 
to cube root of cell volume. The $C_{k}$ and $\mathrm{C}_{c}$ are model constants determined dynamically as developed by Kim and Menon [24]. For the uRANS solution, the two-equation turbulence model, k-omega Shear Stress Transport of Menter [25] is used. The momentum equation in tensorial notation is defined as:

$$
\rho \frac{\partial\left\langle u_{i}\right\rangle}{\partial t}+\rho\left\langle u_{i}\right\rangle \frac{\partial\left\langle u_{i}\right\rangle}{\partial x_{j}}=-\frac{\partial\langle p\rangle}{\partial x_{j}}+\mu \frac{\partial^{2}\left\langle u_{i}\right\rangle}{\partial x_{j}^{2}}-\rho \frac{\partial\left\langle u_{i}^{\prime} u_{j}^{\prime}\right\rangle}{\partial x_{j}}
$$

The bracketed quantities in the equation are phase averaged. The turbulent stresses are modelled as:

$$
\left\langle u_{i}^{\prime} u_{j}^{\prime}\right\rangle=-v_{t r}\left(\frac{\partial\left\langle u_{i}\right\rangle}{\partial x_{j}}+\frac{\partial\left\langle u_{j}\right\rangle}{\partial x_{i}}\right)+\frac{2}{3} k \delta_{i j}
$$

The eddy viscosity $V_{t r}$ is defined by Menter [25]; the formulation allows flow variables to be integrated all over the domain without the need for wall function (low Reynolds number formulation). Such transient form of RANS modelling will result in phase averaged flow velocities that will be used to obtain the now resolved periodic Reynolds stresses. This should not be confused with the modelled stresses computed by Vtr.

The ALE technique is based on that a minimum and maximum cell size and a maximum shape deformation (skewness) are defined in the dynamic mesh zone. Details on the ALE realization is found in [9]; the continuity and momentum equations are modified to account for the deformation of the control volumes. In this study a limit is set that adopts $60 \%$ allowable deviation for the cell shape from an equilateral triangle. The minimum cell size is $0.5 \%$ of the cylinder diameter while the maximum allowed cell size is $0.7 \%$ of the cylinder diameter.

Numerically, the code uses an Euler integration to update the location of the cylinder as in the following equation: $x_{c . g}^{m+1}=x_{c . g}^{m}+v_{c . g} \delta t$. The vector $x_{c . g}$ indicates the position of the cylinder centre of gravity while $v_{c . g}$ indicates its velocity. With a single degree-of-freedom, the cylinder position is solved for the transverse direction. A fourth order Runge-Kutta integration scheme is used to solve the cylinder equation of motion with constant coefficients. The $m_{c y l}, c$ and $k_{s}$ are the cylinder mass, damping and stiffness coefficients respectively as used in the equation of motion:

$$
m_{c y l .} \ddot{y}+c \dot{y}+k_{s} y=F_{\text {fluid-y-direction }}
$$

The effect of the added mass results from the solution of the cylinder-flow coupled system and is not part of the actual mass in the cylinder's equation of motion. Parameters for identifying the structural properties are as follows:

- Specific mass: $\mathrm{m}^{*}=4 m_{c y l} /=\left(\pi D^{2} \rho_{\text {fluid }} l\right)$, where 1 is the cylinder's length.

Mass damping: $m^{*} \zeta, \zeta$ is the damping ratio that is re- lated to the cylinder's damping coefficient by the expression: $c=2 \varsigma \sqrt{k_{s} m_{c y l}}$

The non-linear coupling between the cylinder and the flow field is under taken sequentially. The fluid pressure and shear forces are integrated around the cylinder's surface after a converged solution. Forces are then used to solve the cylinder's equation of motion; displacement is calculated for the next time step. The mesh is deformed and a new solution loop is made. Being chosen to be three orders of magnitude less than the cylinder's oscillating period, the time step size provides stable and accurate coupling between the cylinder surface motion and the flow field.

\section{Results and Discussion}

Simulations are compared against lab-based measurements of Brankovic and Bearman [19] and Hover, et al. [20] with structural properties being of the former. For the experiment of Brankovic and Bearman, $m^{*}$ was 0.82 while $\zeta$ was $1.5 \mathrm{e}^{-4}$. Whereas for Hover, et al. [20], $m^{*}$ was 3.07 and $\zeta$ was 0.013 ; this has an order of magnitude higher specific mass but is still considered as low mass-damping system that is valid for some aspects of comparison. In the referenced experiments, the Reynolds number was about 4000 ; this is one major difference to what is presented herein.

However, before investigating the nature of the FIV predictions, a comprehensive study for a stationary cylinder is presented here for two reasons. First, to establish credible grid sensitivity excluding the influence of the dynamic meshing. Second, as the literature does not provide FIV-Wake details for the studied Reynolds number, the static cylinder comparisons remain essential in judging the quality of the FIV Wake results herein.

A noteworthy reservation herein is that the present study only considers a single reduced velocity in the 'initial regime'. The current study focuses on the method of investigation and what it suggests of interesting flow physics at the low amplitudes occurring in the 'initial regime'. On a figure presentation matter, for the results shown for wake cross-sections at different $x / D$, the curves are shifted against each other for clarity and compactness. The amount of shift varies and equals the difference in the Y-axis variable taken at the minimum $y / D$ value. The shift at the minimum $y / D$ is referenced to either a value of 1 (as for $U / U_{i n}$ ) or a value of zero as for the rest of the plotted variables. Normalization of results is based on the free-stream velocity, diameter of cylinder and working fluid properties.

\section{Static cylinder analysis}

Grid sensitivity studies are shown in Table 2. Grids $R$ are made for $u R A N S 2 D$ studies while for LES studies, a 
Table 2: Parametric comparison of grid sensitivity. Experiments $A$ and $B$ refer to references [8] and [18] respectively. The experimental range is taken from reference [10] except for $\overline{C_{d}}$ that is from [8]. Values are not corrected for experimental setup.

\begin{tabular}{|l|l|l|l|l|l|l|l|}
\hline Grid & $\overline{C_{d}}$ & $C_{d}^{\prime}$ & $-C_{\text {pbase }}$ & $\theta_{s}^{0}$ & $L_{\text {rec }} / D$ & $C_{l}^{\prime}$ & $S t$ \\
\hline$R 1$ & 1.1 & 0.1 & 1.37 & 85.4 & 1.06 & 1.0 & 0.23 \\
\hline$R 2$ & 1.25 & 0.1 & 1.5 & 91 & 1.1 & 1.32 & 0238 \\
\hline$R 3$ & 0.94 & 0.123 & 1.25 & 90 & 1.1 & 0.92 & 0.27 \\
\hline$R 4$ & 0.86 & 0.042 & 1.0 & 84.4 & 1.49 & 0.402 & 0.25 \\
\hline R5 & 0.86 & 0.069 & 1.1 & 85.7 & 1.35 & 0.545 & 0.255 \\
\hline Coarse LES & 0.9 & 0.07 & 1.1 & 87.1 & 1.57 & 0.35 & 0.23 \\
\hline Coarse [7] & 1.12 & 0.07 & 1.23 & 83.81 & 1.432 & 0.37 & 0.206 \\
\hline Fine [7] & 1.454 & - & 1.398 & 96.37 & 1.072 & - & 0.204 \\
\hline Exp. A & 1.237 & - & 1.764 & 95.0 & 0.836 & - & 0.204 \\
\hline Exp. B & 1.44 & - & 1.7 & - & 1.1 & - & 0.179 \\
\hline Range (Exp) & $0.85 \sim 1.45$ & $\approx 0.04$ & - & $\approx 80$ & - & - & 0.21 \\
\hline
\end{tabular}

coarse and a fine grids are computed. Comparisons with lab-based experiments are made against the results of Cantwell and Coles [8] taken by hot wires and Perrin, et al. [18] taken by Particle Image Velocimetry (PIV). The span-to-diameter ratios, blockage ratios and inlet turbulence intensities are $(25,4 \%, 0.6 \%)$ and $(4.8,20.8 \%, 1.5 \%)$ in both experiments respectively. The experimental configurations of both experiments are primarily responsible for their differences as discussed by Perrin, et al. [18]. Moreover, it is important to acknowledge the fact that comparing two experiments that differ significantly in the blockage ratio is a critical matter. In fact, Perrin, et al. [18] compared cautiously their results against that of Cantwell and Coles.

As far as alternative benchmark candidates are concerned, there are pioneering good sources of numerical data from a DNS attempt of Dong and Karniadakis [6] at Reynolds number of 10,000, and a LES study of Breuer [7] at exactly the same Reynolds number studied herein. However, the data presented at each of those numerical benchmarks are limited compared to what is offered in the experimental benchmarks above mentioned. Due to the similarity in Reynolds number, reference to the results of Breuer [7] will be made when applicable; data in Table 2 is for grids using dynamic SGS model [7].

It is shown that the flow variables are very sensitive to grid resolution and that insufficient circumferential grid points result in significant differences in all the reported shedding and wake parameters. The linear eddy viscosity model as applied in the $u R A N S$ method assumes that turbulence generation is in phase with strain rate; hence, a high strain rate downstream of the stagnation point produces an unphysical turbulent viscosity which in turn delays the separation. A consistent over-prediction of the Strouhal number (St) in the $u R A N S$ runs is related to the over-prediction of the separation angle, on the one hand, and to the absence of shear layer transition dictated by the turbulence model that assumes a fully turbu- lent shear layer. In addition, the assumed perfect spanwise correlation owing to the $2 \mathrm{D}$ assumption an effects the shedding phenomenon. Subsequently, the uRANS lift force -which causes the cylinder's motion in this work-, is over predicted. However, its value here should be compared to sectional lift values not overall values reported in Table 2; for that overall values depend on span length.

In light of the $2 \mathrm{D}$ assumption, grids $R 1$ and $R 2$ show reasonable values for forces and vortex frequency. Other $R$ grids over predict the St. Although other $R$ grids display low values for lift force and high values of base pressure that are incidentally closer to experimental observations-, such results do not agree with the perfect span wise correlation assumption that reasonably leads to higher lift force and lower base pressure. It appears that boundary layer resolution is crucial for enhanced predictions of forces and vortex shedding. Perhaps what is controversial here is that $R 1$ grid shows better global quantities than $R 2$ grid which has more than twice as much nodes around the circumference. As the difference between $R 1$ and $R 2$ is not drastic, grid $R 1$ is chosen for uRANS tests for FIV.

The $L E S$ grids show a better force and shedding values from a flow-physical standpoint compared to the assumptions made with the $u R A N S$ solution. Both LES grids show an over prediction in $\mathrm{St}, L_{\text {rec }}$ and $\theta_{s}^{o}$. Although it falls within the experimental range, $\frac{r e c}{C}_{d}$ also does not match the specific available benchmarks but is enhanced by finer grid resolution as also found by Breuer [7]. The coexistence of high St, low mean drag and longer $L_{\text {rec }}$ has been reported by Travin, et al. [26] in their coarse Detached Eddy Simulation study. An important factor that has to be considered herein is the numerical blockage that likely caused a high velocity in shear layers resulting in disagreements in vortex frequency and force predictions.

It is fair to acknowledge that current $L E S$ predictions show different results than that of Breuer [7] perhaps due 


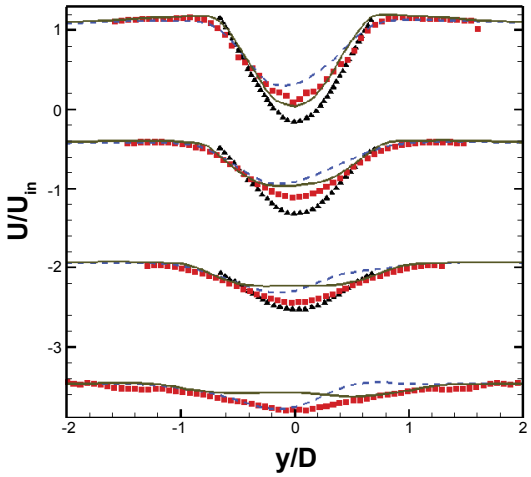

(a)

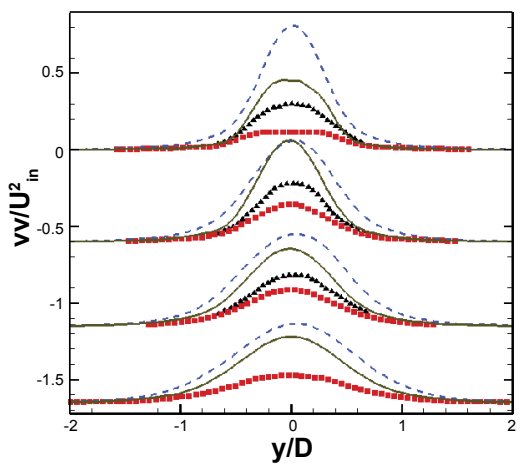

(d)

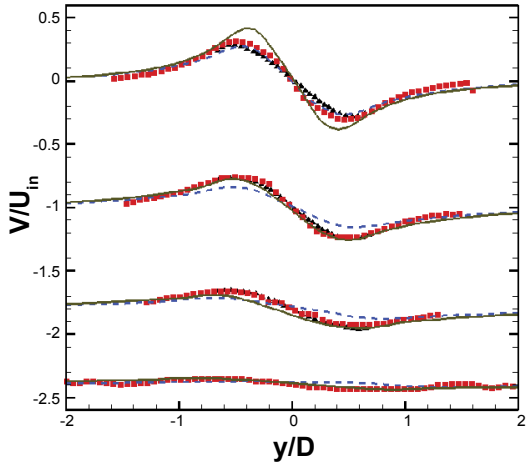

(b)

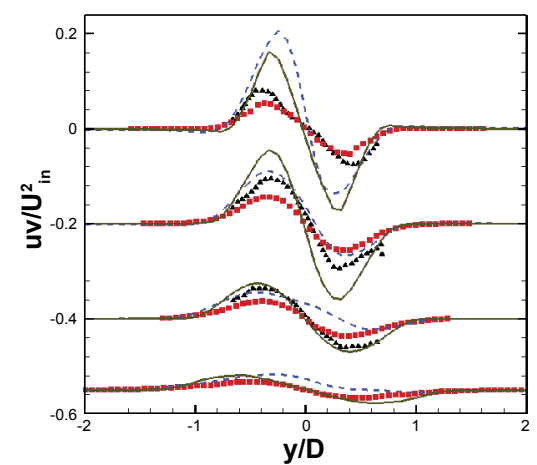

(e)

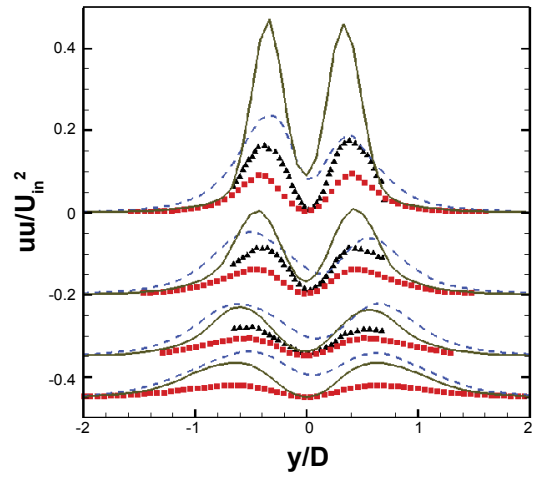

(c)

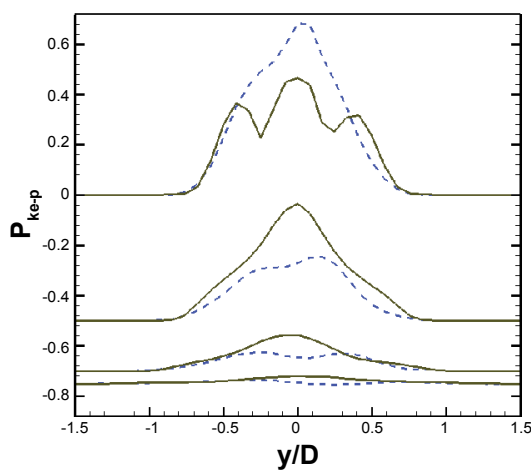

(f)

Figures 2: URANS normalized time averaged velocities, periodic Reynolds stresses $\overline{\tilde{u}_{i} \tilde{u}_{j}}$ and kinetic energy production. Wake cross sections at $x / D \mathrm{x} / D=1.1,1.5,2.0,3.0$ from top to bottom respectively. Square symbols are from [8]; Triangles are from [18]; solid lines are for the static simulation; dashed lines are for FIV simulation.

to their better span wise grid resolution and the computational geometry. However, pro table use could well be made of the current LES studies in order to obtain reasonable insights into FIV interaction with the cylinder's vicinity. Despite its performance, the coarse LES grid will be used for further static and FIV flow comparisons. While the use of the coarse grid would remove some computational burden, evidences from literature are that FIV in 'initial regime' would act to enhance the spanwise correlation requiring less span wise resolution. For quality assessment of $L E S$, the coarse grid shows on Figure $1 \mathrm{~b}$ that modelled turbulence is reaching a maximum of $24 \%$ of the turbulence spectrum at certain spots at the very near wake.

The time averaged velocities for uRANS simulations are displayed as compared to the measurements of benchmark experiments in Figure $2 \mathrm{a}$ and Figure $2 \mathrm{~b}$. The predicted mean stream wise and transverse velocity profiles are in general trend accord with the benchmark measurements. In Figure $3 \mathrm{a}$ and Figure 3b, LES predictions of mean velocities are shown exhibiting similar accord with benchmarks. LES prediction shows more of stream wise velocity deficit along wake profiles due to the difference in computing the recirculation zone.

The turbulent and periodic wake flow around a blue body could be decomposed into three velocity compo- nents as first proposed by Reynolds and Hussain [21]. For an arbitrary instantaneous quantity $s$, its decomposition involves -in order- a global average, a phase (periodic) average and an instantaneous or random component.

$$
s(x, y, t)=\bar{s}+\tilde{s}+s^{\prime}
$$

The sum of the periodic and random components is termed total.

For the benchmark data presented herein, each of the benchmark experiments relied on conditional sampling that is influenced by "phase jitter". As noted by Cantwell and Coles [8] and with more details by Perrin, et al. [18], the conditional sampling results in an under estimation of the coherent/periodic contribution in turbulent stresses. It could be inferred from a Proper Orthogonal Decomposition (POD) study, by Perrin, et al. [27] that up to $40 \%$ under-estimation and $40 \%$ over-estimation can result in the periodic and random turbulence respectively. As the authors do not have the complete POD data of Perrin, et al. [27], all the benchmark data herein are taken by conditional sampling as described in [8] and [18]. In this study, the random contribution is not quantifiable by virtue of the $u R A N S$ assumption adopted. The uRANS output will be compared against periodic turbulence, while the LES output will be compared against the total turbulence. 

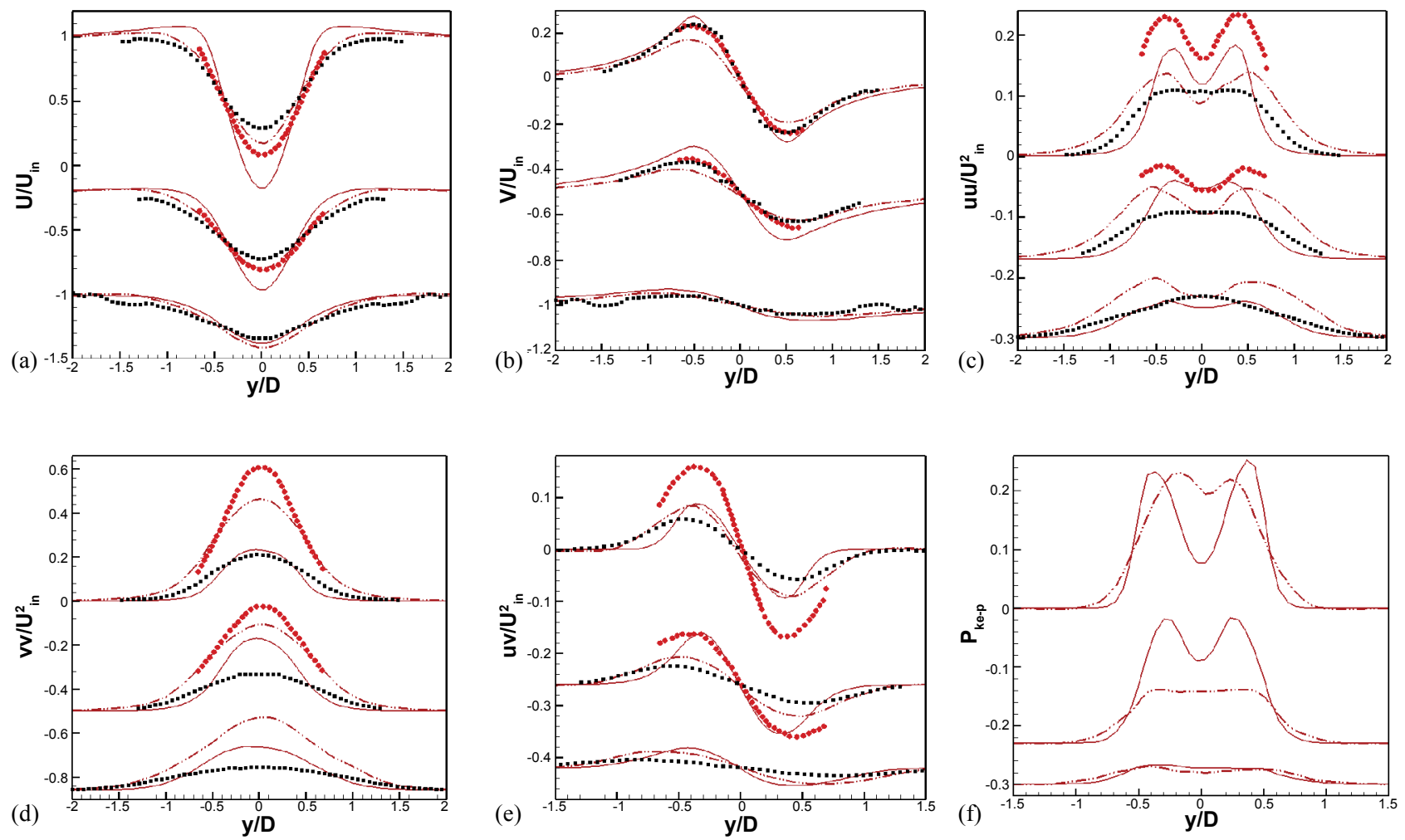

Figures 3: LES normalized time averaged velocities, total Reynolds stresses $\overline{u_{i} u_{j}}$ and kinetic energy production. Wake cross sections at $x / D=1.5,2.0,3.0$ from top to bottom respectively. Square symbols are from (Cantwell and Coles, 1983); Circles are from (Perrin, et al. 2006); solid lines are for the static simulation; dashed lines are for FIV simulation.

The approach to identify phase averaged or periodic contribution relies on obtaining the global average over reasonable shedding cycles- 21 cycles in this study. Global averaged values are subtracted from the instantaneous values of the flow field to yield the periodic component; this was performed over 11 shedding cycles. In this context, the smaller the incremental time step the more resolved the periodic motion. However, there is an upper limit for this very large eddy simulation -applied in $a$ uRANS sense- in that the resolution is tied to the local grid density; hence the small incremental time step used herein to accommodate this requirement. To the knowledge of the authors, such scale decomposition on uRANS has not been looked at in detail for cross cylinder flows at high Reynolds numbers. As for LES computation, time averaging was applied over 25 shedding cycles, with further 25 cycles to obtain converged statistics. Reynolds stresses predicted by LES in the current study are the resolved or filtered in context of the SGS filter applied herein.

In Figure $2 \mathrm{c}$ and Figure $2 \mathrm{~d}$, a general trend between benchmark experiments is that measurements of Perrin, et al. [18] show greater normal periodic stresses than those taken by Cantwell and Coles [8] due to the higher turbulence intensity [18]. The stream wise $\overline{\tilde{u} \tilde{u}}$ and transverse $\tilde{\boldsymbol{v}} \tilde{\boldsymbol{v}}$ periodic stresses are consistently over-predicted, while keeping qualitative accord. Due to the over-predicted St as shown in Table 2, the predicted uRANS periodic normal stresses are high, due to stronger shedding and high velocity in shear layers provided by the numerical blockage. Similarly, the periodic shear stress $\tilde{\boldsymbol{v}} \tilde{\boldsymbol{v}}$ exhibits over-prediction but is in trend wise accord with benchmark counterparts.

Figure $3 \mathrm{c}$ and Figure $3 \mathrm{~d}$ show proles of normal total stresses as predicted by LES method.

The streamwise stresses follow the trend found by Perrin, et al. [18], due to their high blockage ratio and longer Lrec [18]. Accord is enhanced at 2.0D, perhaps due to the higher effect of SGS viscosity upstream that may have decreased the spreading of $\overline{u u}$ across the wake. At $1.5 \mathrm{D}$, transverse stresses approach their counterpart of Cantwell and Coles [8], while being under-predicted compared to data of Perrin, et al. [18]. Better agreement, however, is shown at 2.0D. Taking into account the longer Lrec, it is noteworthy to indicate that such spatial difference in the wake certainly a effects the matching of various variables.

Owing to the difference in blockage ratio, current LES total stresses predictions would be lower than those of Perrin, et al. [18] but would also be higher than the unblocked results of Cantwell and Coles [8]. This overprediction of $\overline{v v}$ has been also reported by Breuer [7] in their LES study. Total shear stress, $\overline{u v}$, is generally higher 
Table 3: Comparison of frequency, forces and separation angle for the Static and FIV studies.

\begin{tabular}{|l|l|l|l|l|l|}
\hline & Fshed, fosc & $\overline{C_{d}}$ & $C_{d}^{\prime}$ & $C_{l}^{\prime}$ & $\theta_{s}^{0}$ \\
\hline (uRANS) Static cyl. & 327 & 1.1 & 0.1 & 1.0 & 85.4 \\
\hline (LES) Static cyl. & 334 & 0.9 & 0.07 & 0.35 & 87.2 \\
\hline (uRANS) FIV cyl. & 325 & 1.44 & 0.58 & 2.14 & 94.53 \\
\hline (LES) FIV cyl. & 287 & 1.52 & 0.23 & 1.7 & 100.1 \\
\hline
\end{tabular}
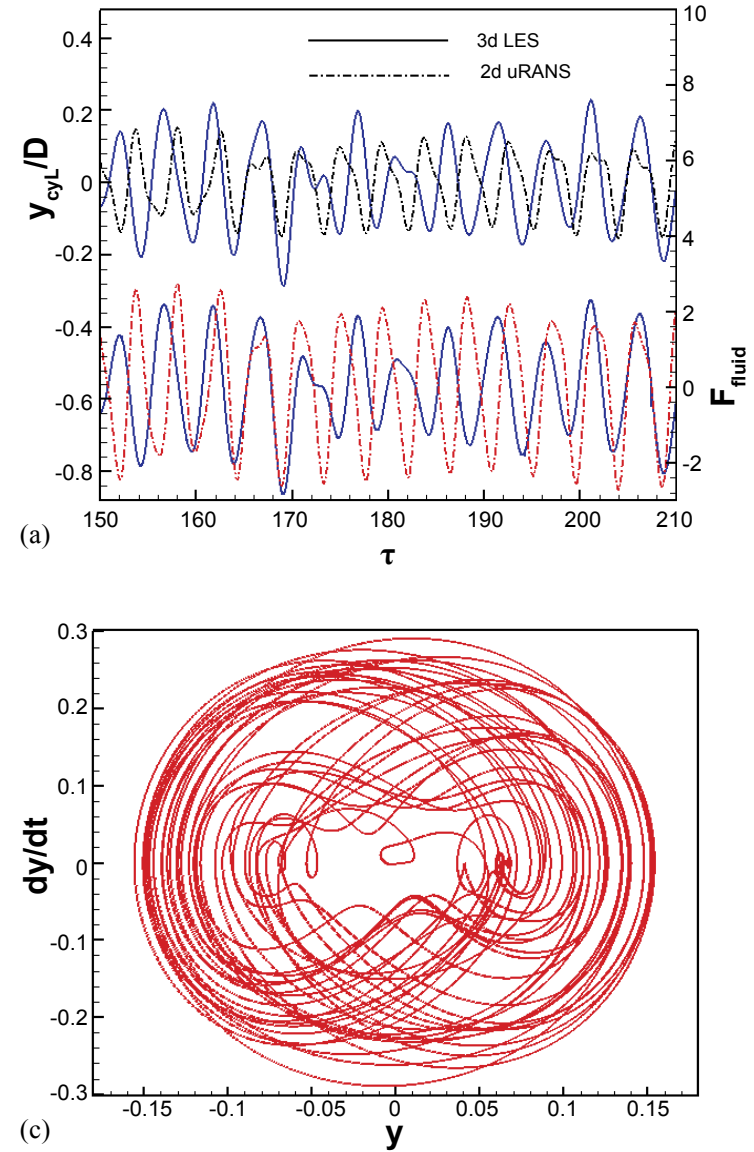
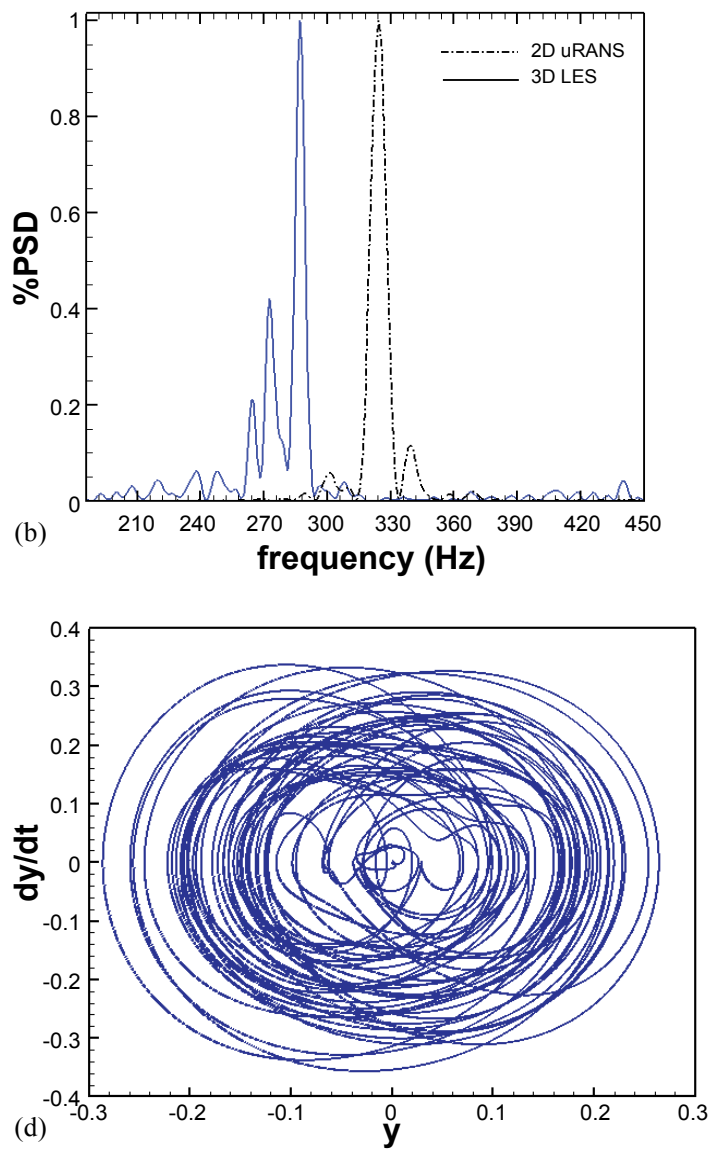

Figures 4: FIV response character a) Normalized lift force and displacement; b) Frequency Spectrum of transverse displacement; c) Phase-space portrait for the uRANS run; d) Phase-space portrait for the LES run.

than that of Cantwell and Coles [8]. However, it is higher at $2.0 \mathrm{D}$ than at $1.5 \mathrm{D}$ due to the difference in recirculation zone Lrec mentioned earlier. Despite the difficulty in simulating the correct physics of a non-equilibrium three-dimensional turbulent wake, the predicted quantities of the flow field fairly suggest a promising attempt that serves the purpose of this work.

\section{Coupled response characteristics}

In the present study, the reduced velocity $V_{r}$ for the cylinder in the $2 \mathrm{D} u R A N S$ run and the $3 \mathrm{D} L E S$ runs was 1.5 and 2 respectively. Due to the over-estimated lift force in the $2 \mathrm{D} u R A N S$, a reduced velocity of 2 has resulted in an increased displacement amplitude and a $2 \mathrm{P}$ shedding pattern. Hence, either choice of $V_{r}$ meant that the simulation takes place in the 'initial regime' with a shedding pattern of $2 \mathrm{~S}$. Table 3 shows the effect of the flow induced vibration on the shedding frequency, lift and drag forces.
The shedding frequency reported in the Table 3 is obtained by examining the frequency of the velocity trace in the downstream wake. The oscillating cylinder excited by the flow vortex force shows a dominant response frequency that is now the system's frequency (i.e. the wake and the cylinder together). Figure $4 \mathrm{a}$ and Figure $4 \mathrm{~b}$ show the response curves for both lift force and transverse distance moved by the cylinder along with the frequency spectrum of the signals.

It could be inferred that, in $u R A N S$ and LES responses, the signal is not periodic (non-stationary) and that LES response is more of chaotic. Evidence of this can be found by inspecting the phase-space profiles in Figure $4 \mathrm{c}$ and Figure $4 \mathrm{~d}$, where multiple interfering orbits are present. The effect of Reynolds number is shown by the higher and more chaotic response compared to response spectrums in Brankovic and Bearman [19], and a CFD study by Elbanhawy and Turan [9], both undertaken at Reynolds number of around 4000. 
Table 4: Characteristics of flow induced vibration response for the different studied cases compared to benchmarks. The arrow sign denotes the range of the measured quantity.

\begin{tabular}{|l|l|l|l|l|l|}
\hline & $Y_{\text {cyll }}$ (D & $\Delta \Phi^{0}$ & fosc/fn & $C_{l a}$ & $C_{\text {lu }}$ \\
\hline Hover, et al. [20] & 0.02 & $-20 \rightarrow 25$ & 0.40 & $-0.3 \rightarrow-0.8$ & $-0.8 \rightarrow 0.15$ \\
\hline Brankovic and Bearman [19] & 0.13 & 0.0 & 0.47 & -0.9 & -0.02 \\
\hline (LES) FIV run & 0.2 & 0.0 & 0.41 & -1.47 & $2.8 e^{-6}$ \\
\hline (uRANS) FIV run & 0.11 & 0.0 & 0.46 & -1.64 & 0.0021 \\
\hline Elbanhawy and Turan [9] & 0.07 & 0.0 & 0.45 & -0.7 & $8.3 e^{-4}$ \\
\hline
\end{tabular}

The frequency spectrum here is analysed using Fast Fourier Transform algorithm with a Tukey window applied in Matlab [28]. A Nyquist frequency of $250,000 \mathrm{~Hz}$, which is three orders of magnitude higher than the system's frequency, is used for sampling. The oscillating cylinder exhibits a significant amplification of fluctuation of drag and lift forces -as expected-in both $u R A N S$ and LES runs. However, the LES run shows more sensitivity to FIV with an order of magnitude rise in force fluctuations. With FIV, the shedding frequency of the flow-excited cylinder has decreased slightly but remained matched to the cylinder's oscillating frequency.

In accordance with either of the benchmark experimental measurements, the phase angle in between the fluid force and the cylinder's displacement is zero as indicated in Table 4. The force coefficients along with frequency ratio and vertical amplitude are shown in Table 4 . The force coefficients - where $N$ denotes the number of discrete sample points- are calculated by means of correlation functions between angle brackets as follows:

$$
\begin{aligned}
& C_{l v}=\sqrt{\frac{2}{N}} \frac{\langle F(t), \dot{y}(t)\rangle}{\sqrt{\langle\dot{y}(t), \dot{y}(t)\rangle}} \frac{1}{0.5 \rho l D U_{i n}^{2}} \\
& C_{l a}=\sqrt{\frac{2}{N}} \frac{\langle F(t), \dot{y}(t)\rangle}{\sqrt{\langle\ddot{y}(t), \ddot{y}(t)\rangle} \frac{1}{0.5 \rho l D U_{i n}^{2}}}
\end{aligned}
$$

The numerical predictions for the oscillating cylinder show an increase in the displacement amplitude and a striking difference in the $C_{l a}$. The sign of the added mass coefficient from Brankovic and Bearman [19] has been altered to be compatible with the same method of correlation applied here and in Hover, et al. [20]; this implies that when the $C_{l a}$ is negative, the added mass is positive. Other parameters show accord with the range of experimental results laid out in Table 4; it is noteworthy that both experimental values are for the 'initial regime' with very similar structural properties. CFD predictions by Elbanhawy and Turan [9] at a lower Reynolds number likely suggest that the increased displacement and added mass effect on the force $\left(C_{l a}\right)$ are Reynolds number effects.

\section{Wake dynamics with FIV}

FIV influence on wake profile is shown on Figure 2 for the $u R A N S$ run and in Figure 3 for the LES run. It seems that the averaging time was not long enough for the uRANS FIV run as indicated by the slightly unsymmetrical wake profiles. The cylinder's oscillations seem to reduce the stream wise velocity deficit across the wake, especially in the vicinity of the cylinder as seen on Figure $2 \mathrm{a}$ and Figure 3a. However, both $u R A N S$ and LES runs show that such deficit increases further downstream of the cylinder and becomes higher than the static case, due to the expected mixing of free stream and Vortex Street. This influence on the recirculation zone is consistent with what is found in Govardhan and Williamson [11], whereas what is not consistent with their measurements is that they observed a higher velocity deficit in the 'initial regime' marked with $2 S$ Karman vortex street shedding. The non-harmonic response predicted here would result in a slightly disturbed $2 S$ shedding regime; this consequently leads to a different wake average field compared to Govardhan and Williamson [11] given that their experiment was made at Reynolds number of 4000.

Oscillations have consistently reduced the average transverse velocity along the wake as inferred from Figure $2 \mathrm{~b}$ and Figure $3 \mathrm{~b}$. Such decrease is more pronounced at the vicinity of the cylinder than further downstream. This is reasonable given the degree of wake mixing expected by the spatial increase of the vortex origins on both sides of the cylinder due to vibration. The cylinder's oscillations have widened the effect of the wake as appears on the stream wise, transverse and shear periodic stresses in Figure $2 c$ and their LES total counterparts in Figure $3 \mathrm{c}$. This agrees with measurements of Govardhan and Williamson [11] for a lower Reynolds number.

FIV causes periodic and total stream wise stresses to increase while moving downstream, although it causes a decrease at 1.1D and 1.5D. As expected, due to the cross-flow oscillations that organise vortex motion, both transverse periodic and total stresses are increased at the different wake cross-sections shown in Figure $2 d$ and Figure $3 \mathrm{~d}$. The periodic and total shear stress appears to be influenced by FIV and its free stream entrainment and is thus decreased along the wake as seen in Figure 2e and Figure 3e. This is not the case for the uRANS result at $1.1 \mathrm{D}$ where entrainment is not fully achieved, provided that $u R A N S$ prediction consistently over predicts the $p e-$ riodic shear stress. In their study, Govardhan and Wil- 
liamson [11] have also observed that FIV reduces total and periodic shear stress at a lower Reynolds number.

The time averaged kinetic energy production of vortex structures is evaluated from the main stream to the resolved motion as follows:

$$
P_{(k e-p)}=-\overline{u_{i} u_{j}} \frac{\partial U_{i}}{\partial x_{j}}
$$

This is the turbulence energy production determined from the mean flow gradients and the resolved Reynolds stresses $\overline{u_{i} u_{j}}$ whether these stresses are the periodic (uRANS) or the filtered (LES). Time averaged turbulent energy production along wake cross-sections is displayed in Figures $2 \mathrm{f}$ and Figure $3 \mathrm{f}$ showing the influence of FIV. Except at 1.1D for the $u R A N S$ run, as the distance increases downstream, FIV results in decreased production; that is suggested to be due to decreased flow gradients resulting from wake mixing. As the shear plays an important role in such production, this uRANS observation at $1.1 \mathrm{D}$ is expected.

There is an apparent difference in the production profile between the $u R A N S$ and LES runs owing to the difference in turbulence treatment and more likely to that the $u$ RANS solution does not possess a span dimension. However, with the presence of FIV, production profiles share the same trend in both of the uRANS and LES runs. A general trend shown is that the production of coherent structures is enhanced near the cylinder's base where the recirculation zone is. This is true for both static and glow-excited cylinder cases presented herein given the difference in their respective recirculation zones. This is clearly seen for a static cylinder by Cantwell and Coles [8] although not available to be shown herein.

While the wake plots of turbulent energy production seem to follow the same trend among the static and FIV cases, the qualitative and quantitative distribution of vortex energy and its production differ significantly nearer the cylinder's boundary layer. To further the analysis, Figure $5 \mathrm{a}$ visualises the $u$ RANS kinetic energy production in the vicinity of the cylinder. For the static case, it is shown that the thin boundary layer has positive energy, the maximum of which lies in the separated shear layer, delivered to augment the coherent eddy structures. The base of the cylinder has a region of negative production zone that serves as a sink for the energy of the periodic structures.

On the contrary, the FIV case exhibits major differences in the profile of energy production Figure $5 b$. The thin boundary layer area is almost dominated by negative production (decrease of energy of coherent structures). However, away from the thin boundary layer area, there is a region of strong energy production as part of the separated shear layer and in the base of the cylinder. The core of the recirculation zone shows a different behaviour in between the static and the oscillating cylinder. Irrespective of its sign (i.e. direction), kinetic energy production is consistently higher -about one order of magnitude- influenced by the cylinder's oscillation.

Alternatively, Figure 6a visualises the LES kinetic energy production in the vicinity of the cylinder where values are generally less than those predicted by $u R A N S$. FIV causes an increase in production by more than an order of magnitude in addition to a qualitative difference in production profile. Generally, uRANS and LES runs share the same influence of FIV on production values although there remains a difference in magnitudes where the influence of FIV is higher in LES computations.
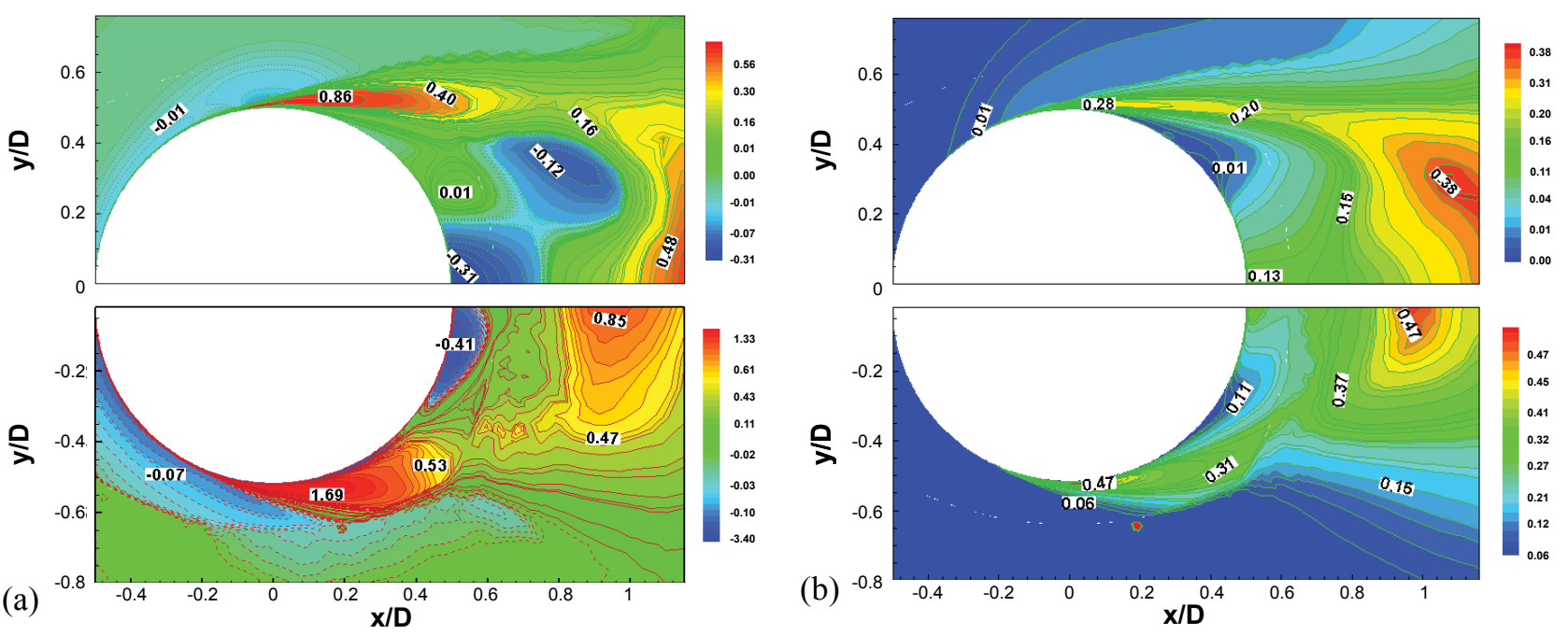

Figures 5: uRANS normalized time-averaged energy data for coherent structures a) Kinetic energy production contours where dashed lines denote negative values and dark regions indicate higher absolute values, static cylinder at top and FIV cylinder at bottom; b) Kinetic Energy contours of coherent structures, dark regions indicate higher values, static at top and FIV at bottom. 

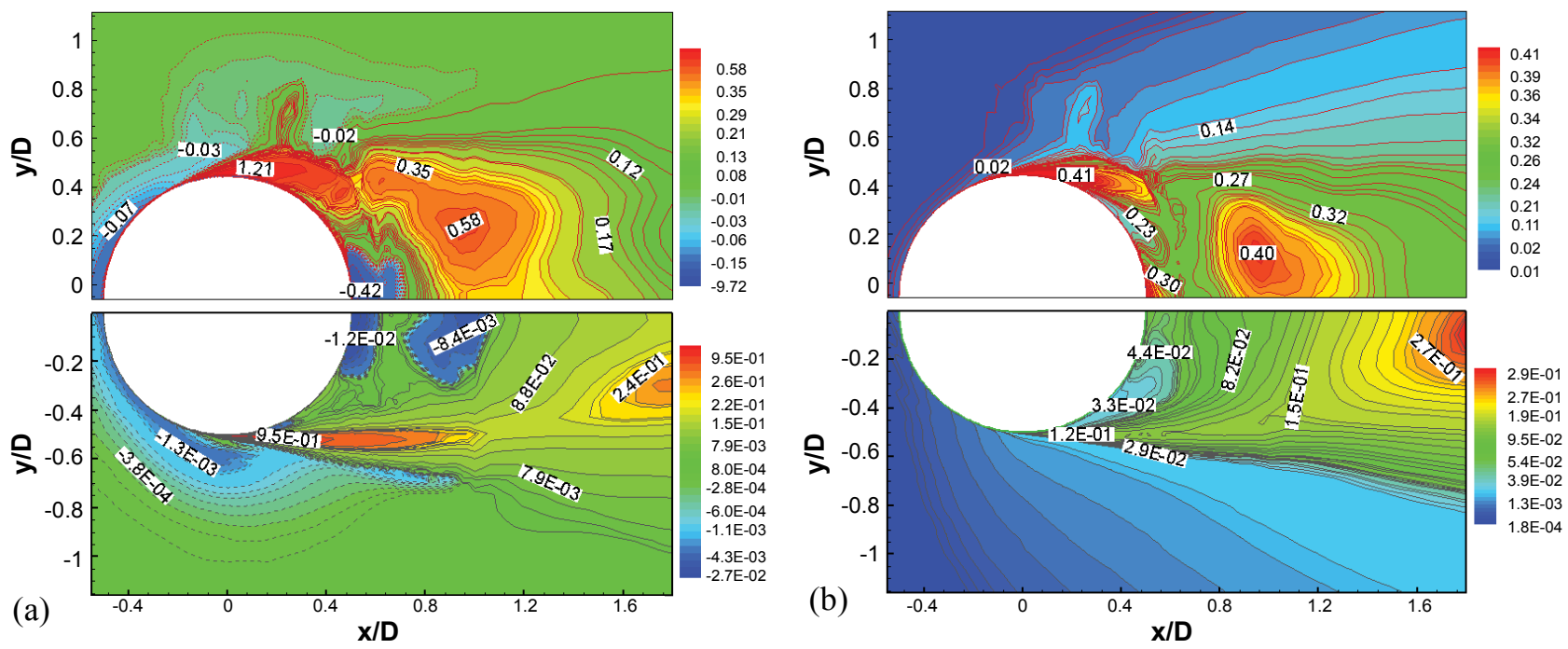

Figures 6: LES normalized time-averaged energy data for coherent structures a) Kinetic energy production contours where dashed lines denote negative values and dark regions indicate higher absolute values, FIV cylinder at top and static cylinder at bottom; b) Kinetic Energy contours of coherent structures, dark regions indicate higher values, FIV cylinder at top and static cylinder at bottom.

Time averaged kinetic energy of resolved vortex structures is visualised in Figure $5 \mathrm{~b}$ and Figure $6 \mathrm{~b}$ respectively for the $u R A N S$ and LES runs. Similar to $P_{(k e-p),}$ the cylinder's oscillation causes a significant increase -by not less than an order of magnitude in the kinetic energy. This increase is more pronounced in the separated shear layers and at the cylinder's base. Both $u R A N S$ and LES runs follow the same trend despite the fact that $u R A N S$ simulation predicts higher time averaged kinetic energy. This is suggested to be due to the two dimensional assumption that concentrates vorticity in a two dimensional plane.

The fluctuation of static pressure and its correlation with stream-wise and transverse velocities on the cylinder surface are plotted in Figure 7. It is shown in Figure 7a that the URANS method over-predicts the time averaged pressure fluctuations on the cylinder surface compared to the LES output where values are influenced by the span dimension. FIV causes such fluctuations to increase considerably; however, values from both $u R A N S$ and LES come closer to one another. Such observation supports the argument that oscillations enhance the two-dimensionality of the cylinder's wake.

Time averaged $p-u$ correlation is generally enhanced by FIV as shown in Figure 7b. However, $u$ RANS method exhibits higher correlation likely due to the higher shear layer stream wise velocity. Such high velocity results from the linear eddy viscosity turbulence model that assumes that production and strain rate are in the same phase. Figure $7 c$ shows time averaged $p-v$ correlation where FIV also increases such correlation and alters the circumferential distribution qualitatively. LES computa- tion shows higher $p$ - $v$ correlation values than its $u R A N S$ counterpart. This observation comes in support to the higher oscillation amplitude given by the LES solution, which in turn, causes higher fluctuations in transverse velocity at the cylinder boundary.

\section{Conclusions}

High Reynolds number $(140,000)$ FIV-Wake interaction studies are undertaken numerically for the first time in the literature. In agreement with previous reviews in the open literature, it is found that FIV cylinder-dynamics exhibit important changes in high Reynolds number flows. The wake of the circular cylinder in the present CFD study has exhibited distinct vortical structures that are amenable to resolution by means of the turbulence treatment applied. Numerical predictions for wake statistics and response characteristics provided reasonable indications to what FIV would lead to.

Results have shown that pro table use could be made out of $2 \mathrm{D}$ uRANS simulations where expensive computational resources are of an issue. While recognizing the level of approximations applied herein, it is shown that the high Reynolds number influences the cylinder's response amplitude, added mass and lift/drag amplification.

Furthermore, FIV enhances free stream entrainment into the wake and results in changes in periodic and total Reynolds stresses. Significant changes in the kinetic energy of vortex structures and its production occur around the cylinder boundary especially in the separated shear layer and at the cylinder base. While the current study covers a certain scenario of cylinder FIV, useful insights on the influence of Reynolds number have been presented. Further research is needed to explore high Reynolds 

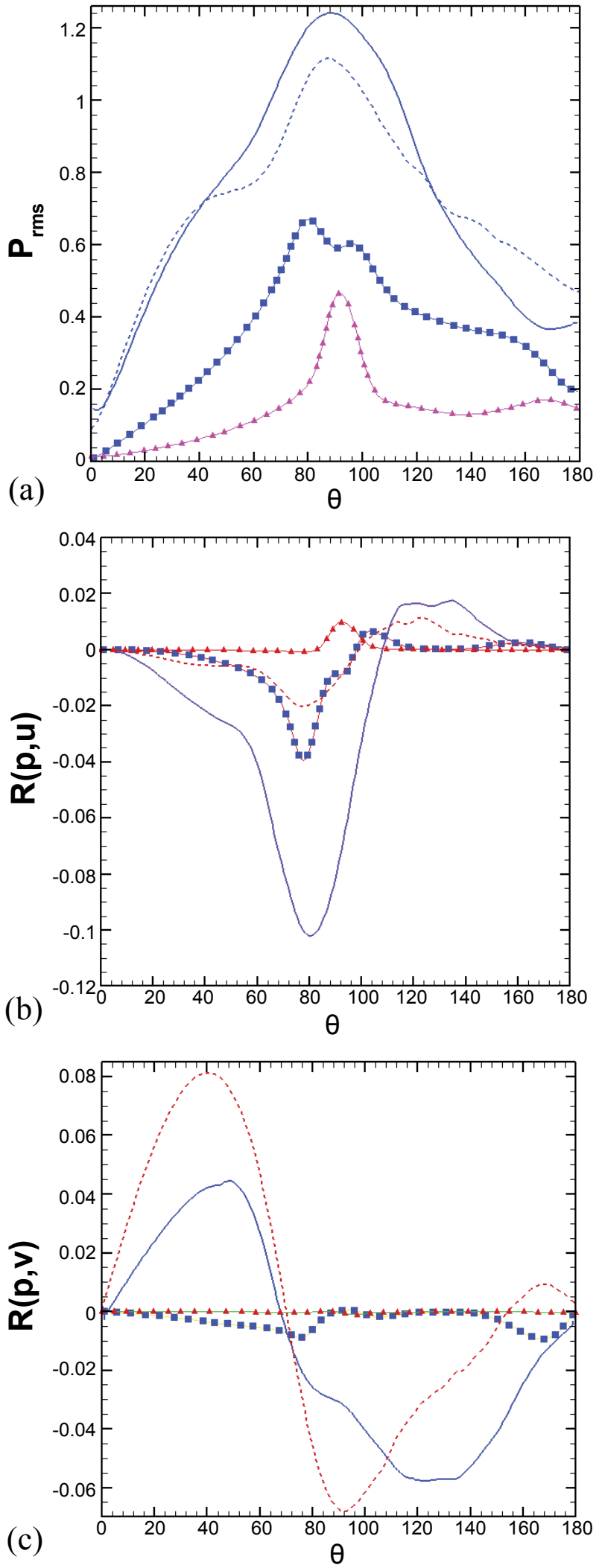

Figures 7: Time averaged data on the upper surface of the cylinder a) Static pressure fluctuation (RMS); b) Correlation of static pressure and streamwise velocity; c) Correlation of static pressure and transverse velocity. Square symbols are for the URANS static run, Triangle symbols are for the LES static runs. Solid lines are for the FIV run (uRANS), Dashed lines are for the FIV run (LES). number effects on other regimes of FIV response while possibly covering different values of the $m^{*} \zeta$ parameter.

\section{Acknowledgement}

The authors would like to acknowledge the research fund from the University of Manchester. Gratitude is due to Dr. A. Revell (University of Manchester) for assistance, and to Prof. M. Braza (IMFT) for the access of experimental data used for benchmarking.

\section{References}

1. T Sarpkaya (2004) A critical review of the intrinsic nature of vortex-induced vibrations. Journal of Fluids and Structures 19: 389-447.

2. CHK Williamson, R Govardhan (2008) A brief review of recent results in vortex induced vibrations. Journal of Wind Engineering and Industrial Aerodynamics 96: 713-735.

3. RD Gabbai, H Benaroya (2005) An overview of modeling and experiments of vortex induced vibration of circular cylinders. Journal of Sound and Vibration 282: 575-616.

4. P Anagnostopoulos (2002) Vibrations induced by vortex shedding. In: P Anagnostopoulos, Flow-Induced Vibrations in Engineering Practice. WIT press, UK, 1-80.

5. RD Blevins (1994) Flow-Induced Vibration. (2 ${ }^{\text {nd }}$ edn), Krieger Publishing Company, Florida.

6. S Dong, GE Karniadakis (2005) DNS of flow past a stationary and oscillating cylinder at $\operatorname{Re}=10000$. Journal of Fluids and Structures 20: 519-531.

7. M Breuer (2000) A challenging test case for a large eddy simulation: high Reynolds number circular cylinder flow. International Journal of Heat and Fluid Flow 20: 648-654.

8. B Cantwell, D Coles (1983) An experimental study of entrainment and transport in the turbulent near wake of a circular cylinder. Journal of Fluid Mechanics 136: 321-374.

9. A Elbanhawy, A Turan (2010) On Two-dimensional Predictions of Turbulent Cross- flow Induced Vibration: Forces on a Cylinder and Wake Interaction. Flow, Turbulence and Combustion 85: 199-224.

10. MM Zdravkovich (1997) Flow around circular cylinders: a comprehensive guide through flow phenomena. 1: Fundamentals, Oxford University Press, UK.

11. R Govardhan, CHK Williamson (2001) Mean and fluctuating velocity fields in the wake of a freely-vibrating cylinder. Journal of Fluids and Structures 15: 489-501.

12. RMC So, Y Zhou, MH Liu (2000) Free vibrations of an elastic cylinder in a cross flow and their effects on the near wake. Experiments in Fluids 29: 130-144.

13. R Gorvardhan, CHK Williamson (2006) Defining the 'modified Griffin Plot' in vortex-induced vibration: revealing the effect of Reynolds number using controlled damping. Journal of Fluid Mechanics 561: 147-180.

14. A Elbanhawy, A Turan (2010) Heat Transfer and Wake Interaction Dynamics for Low Mass-Damping Cylinder Undergoing Flow-Induced Vibration At High Reynolds Number. Proc. ASME $3^{\text {rd }}$ Fluids Engineering Summer Meeting, Montreal, Canada.

15. T Tamura, I Ohta, K Kuwahara (1990) On the reliability of two-dimensional simulation for unsteady flows around a cylinder-type structure. Journal of Wind Engineering and Industrial Aerodynamics 35: 275-298. 
16. Bouhadji, S Bourdet, M Braza, Y Hoarau, P Rodes, et al. (2003) Turbulence modelling of unsteady flows with a pronounced periodic character. In: W Hasse, V Selmin, B Winzell Progress in computational flow-structure interaction. Springer Verlag, Germany, 87-96.

17. (2010) ANSYS Fluent Theory Guide. Canonsburg, PA, USA.

18. R Perrin, M Braza, E Cid, S Cazin, F Moradei, et al. (2006) Near-wake turbulence properties in the high Reynolds number incompressible flow around a circular cylinder measured by two-and three-component PIV. Flow, Turbulence and Combustion 77: 185-204.

19. M Brankovic, PW Bearman (2006) Measurements of transverse forces on circular cylinders undergoing vortex-induced vibration. Journal of Fluids and Structures 22: 829-836.

20. FS Hover, AH Techet, MS Traintafyllou (1998) Forces on oscillating uniform and tapered cylinders in cross flow. Journal of Fluid Mechanics 363: 97-114.

21. WC Reynolds, AKMF Hussain (1972) The mechanics of an organized wave in turbulent shear flow. Part3. Theoretical models and comparisons with experiments. Journal of Fluid Mechanics 54: 263-288.
22. BP Leonard (1991) The Ultimate conservative difference scheme applied to unsteady one-dimensional advection. Journal of Computational Methods in Applied Mechanics and Engineering 88: 17-74.

23. BP Leonard, S Mokhtari (1990) ULTRA-SHARP Nonoscillatory Convection Schemes for High-Speed Steady Multidimensional Flow. NASA Lewis Research Center.

24. WW Kim, S Menon (1997) Application of the localized dynamic subgrid-scale model to turbulent wall-bounded flows. $35^{\text {th }}$ Aerospace Sciences Meeting.

25. FR Menter (1994) Two-equation eddy-viscosity turbulence models for engineering applications. AIAA Journal 32: 1598-1605.

26. Travin, M Shur, M Strelets, P Spalart (1999) Detached-eddy simulations past a circular cylinder. Journal of Flow Turbulence and Combustion 63: 293-313.

27. R Perrin, M Braza, E Cid, S Cazin, A Barthet, et al. (2007) Obtaining phase averaged turbulence properties in the near wake of a circular cylinder at high Reynolds number using POD. Experiments in Fluids 43: 341-355.

28. Matlab (2008) The MathWorks Inc. Natick, MA, USA. 